\title{
Revista de Investigación Educativa 4
}

enero-junio 2007 | ISSN 1870-5308 | Xalapa, Veracruz

Instituto de Investigaciones en Educación | Universidad Veracruzana

\section{Razones por la transparencia en la universidad}

\author{
Miguel Angel Casillas Alvarado \\ Jessica Badillo Guzmán \\ Karla Alejandra Valencia-González Romero \\ Universidad Veracruzana \\ Instituto de Investigaciones en Educación
}

El texto inicia con una serie de anotaciones en torno a las circunstancias institucionales en que surge la necesidad de la transparencia en la Universidad Veracruzana, haciendo hincapié en que la Universidad ha permanecido por años en completa secrecía alrededor de muchos de sus procesos. Posteriormente los autores presentan cuatro razones por la transparencia en la Universidad, mismos que identifican como elementos para exponer los motivos de la reforma universitaria en este ámbito y como objetivos esperados que puedan ser evaluados en el futuro. Dichas razones son: la actualización del marco normativo, la construcción de una nueva manera de ser universitario, la relación entre transparencia, autonomía y rendición de cuentas a la sociedad, así como la contribución de la Universidad a la formación de ciudadanos.

Palabras clave: Universidad Veracruzana, acceso a la información, reforma educativa.

The text initiates with a series of annotations around the institutional circumstances in that the necessity arises from the transparency in the Veracruzana University, insisting on which the University has remained per years in complete secret around many of its processes. Later the authors present four reasons by the transparency in the University, which they identify like elements to expose the reasons for the university reform in this scope and like objectives that can be evaluated in the future. These reasons are: the update of the normativity, the construction of a new way to be a university member, the relationship between transparency, autonomy and accountability, as well as the contribution from the University to the formation of citizens.

Key words: Universidad Veracruzana, information acces, educational reform.

Para citar este artículo:

Casillas, M. A., Badillo, J. \& Valencia-González, K. A. (2007, enero-junio). Razones por la transparencia en la universidad. CPU-e, Revista de Investigación Educativa, 4. Recuperado el [fecha de consulta], de http://www.uv.mx/cpue/num4/critica/casillas_razones_transparencia.htm 


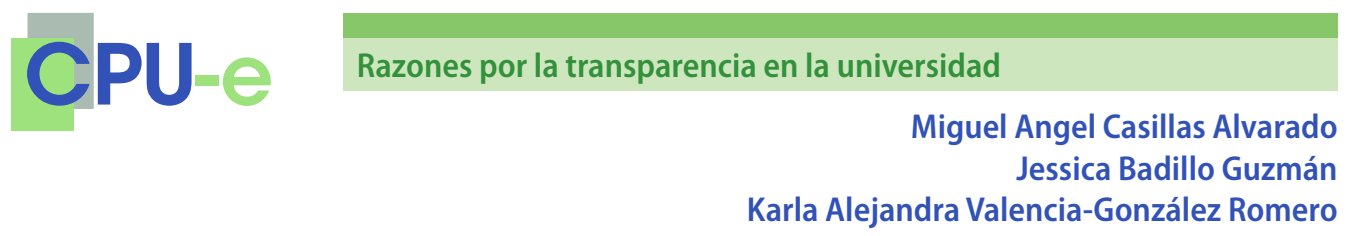

\section{Razones por la transparencia en la universidad}

\section{Introducción}

— $\mathrm{n}$ la Universidad Veracruzana, la iniciativa por abrir su gestión al escrutinio _úblico llegó y se expresó como una posición cohesionada al calor de la sucesión rectoral del $2005 .{ }^{1}$ En aquel momento, un grupo de académicos propuso que se desarrollara un foro para la reforma universitaria; en realidad fueron varias sesiones, pero en la referida al gobierno universitario fue el Dr. José González Sierra quien sugirió que discutiéramos el problema de una ley universitaria de transparencia. Muchas de las aportaciones del foro fueron retomadas en el programa del Rector de la Universidad, Dr. Raúl Arias Lovillo, quien impulsó que se creara un reglamento de transparencia y rendición de cuentas, con carácter innovador y de vanguardia en el medio universitario mexicano.

El compromiso específico de elaborar un proyecto de reglamento de transparencia tuvo un punto de origen que marcó el desarrollo de un proceso participativo de construcción de un consenso institucional.

El punto de origen fue aquella sesión del Consejo Universitario donde públicamente se informó del conflicto patrimonial que afectaba a la Institución por el litigio con un arrendatario de computadoras. Problema mayúsculo por las decenas de millones de pesos involucradas, por ser evidencia de la ineptitud, la ineficacia y la falta de control institucional en el manejo de su patrimonio, así como por estar involucrados distinguidos personajes de la anterior administración universitaria y algunos importantes miembros del actual gobierno del estado de Veracruz. Cuando el Consejo Universitario conoció del problema, apoyó de manera unánime al Rector en el conflicto político-legal, reconoció la necesidad de abordar de una manera institucional el problema y encargó una investigación a su comisión de Honor y Justicia, asumiendo que la Universidad debería transformarse para que estas cosas nunca más se volvieran a repetir.

Precisamente porque nace en el marco de una gestión rectoral que está buscando cómo abrirse a la participación de los universitarios en la conducción

I La iniciativa llegó tarde, no sólo a Veracruz sino a la Universidad Veracruzana. La Ley Federal de Transparencia y Acceso a la Información Pública Gubernamental data de junio de 2002 y los reglamentos de algunas instituciones de educación superior mexicanas se elaboraron desde el año 2003 . 


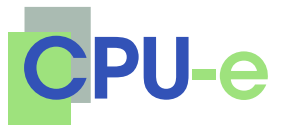

Razones por la transparencia en la universidad

Miguel Angel Casillas Alvarado

Jessica Badillo Guzmán

Karla Alejandra Valencia-González Romero

institucional, el proceso de discusión del reglamento de transparencia debía ser diferente a procesos legislativos anteriores. En este contexto, en octubre de 2006 la Universidad Veracruzana firmó un convenio de colaboración con el Instituto Federal de Acceso a la Información (IFAI) para promover la cultura de transparencia y acceso a la información al interior de la Universidad. Por su parte, la comisión de Reglamentos del Consejo Universitario elaboró una propuesta inicial que fue aprobada en lo general en la sesión del 23 de noviembre de 2006, sesión en la que también el exrector de la uv, Dr. Emilio Gidi Villarreal, fue designado Defensor de los derechos de los universitarios.

A partir de entonces muchas cosas han cambiado. Hacia febrero de 2007, el Congreso del Estado derogó la Ley de Acceso a la Información del Estado de Veracruz de Ignacio de la Llave (publicada en la Gaceta Oficial de fecha 8 de junio de 2004), y aprobó la Ley 848 de Transparencia y Acceso a la Información Pública para el Estado de Veracruz, que incorpora a la Universidad como sujeto obligado en su Artículo 5, Fracción VI. Lo anterior exigía a la Universidad realizar diversas adecuaciones al proyecto de reglamento original.

Así también, se han desarrollado eventos y discusiones que han enriquecido el proyecto y definiendo su sentido innovador; entre ellos sobresale la realización del Encuentro sobre la Transparencia y el Acceso a la Información en las Universidades Públicas, convocado por el Ifai y la Universidad Veracruzana, el pasado mes de marzo. En este evento se dieron cita especialistas en cuestiones de acceso a la información a nivel local, regional y nacional; especial mención merece la participación de académicos especialistas en cuestiones universitarias: Ciro Murayama Rendón, de la Universidad Nacional Autónoma de México; Rollin Kent Serna, de la Benemérita Universidad Autónoma de Puebla y Manuel Gil Antón, por la Universidad Autónoma Metropolitana, quienes hicieron enriquecedoras aportaciones en torno a la transparencia y el acceso a la información en las instituciones de educación superior, tanto públicas como privadas.

Como puede observarse, el proceso de discusión está abierto y estas líneas pretenden ser una invitación a la continuidad en el debate sobre la transparencia.

Quienes redactamos el presente ensayo, hemos colaborado en diferentes momentos con esta iniciativa de transformación legislativa; sin embargo, observamos que en la discusión general, la reflexión que aborda el sentido que actualmente tiene para las instituciones universitarias abrir su gestión y exponer sus resultados a toda la sociedad, sigue siendo muy poco desarrollada. Este documento apunta en esa dirección, al tratar de exponer algunas de las principales razones que nos conducen a la transparencia en la Universidad Veracruzana. 


\section{La transparencia, un corte con el pasado}

La construcción de un régimen político democrático ha sido uno de los rasgos del cambio social más importantes de los últimos años. Su edificación ha sido posible por el agotamiento del régimen político autoritario que caracterizó a los gobiernos del prí́smo (que contaminó con su cultura política a todos los partidos y organizaciones sociales) y por la emergencia de una sociedad civil más participativa y comprometida; su génesis se asocia a las luchas democratizadoras que indígenas, trabajadores, universitarios y un sinfín de organizaciones han establecido en los últimos cincuenta años; sus procesos más significativos han sido las alternancias en distintos órdenes de gobierno, la competencia electoral libre, la defensa del pleno ejercicio de los derechos civiles, el derecho a la información y a la rendición de cuentas de los funcionarios públicos. Sus instituciones emblemáticas son el Instituto Federal Electoral, la Comisión Nacional de Derechos Humanos, el Instituto Federal de Acceso a la Información, la Auditoría Superior de la Federación, la autonomía del Poder Judicial, la pluralidad de las Cámaras.

Todo ello se ha edificado contra la dinámica y la cultura implantadas por el antiguo régimen político autoritario, específicamente respecto de la conducción de las instituciones. De esta manera, la transparencia y la rendición de cuentas son claros instrumentos para dotar a los ciudadanos de información y de poder para luchar en contra del clientelismo, la corrupción, la discrecionalidad, el autoritarismo en las decisiones, el secreto y la confidencialidad burocrática. Son instrumentos que, usados por los ciudadanos, pueden resultar eficientes para controlar los actos del gobierno.

Es muy importante señalar que en nuestra Universidad no hay una tradición de apertura, transparencia y rendición de cuentas. Incluso su Archivo Histórico, que debería ser un espacio privilegiado para la conservación de su memoria institucional, se encuentra fuera de las prioridades institucionales y sus trabajadores hacen milagros con sus escasos recursos y malas condiciones de trabajo. Por su parte, los informes de los rectores, antes que ser instrumentos para la toma de decisiones institucionales, para fomentar el conocimiento de la Universidad, para evaluar los resultados de las gestiones y acciones emprendidas en un plazo determinado, ${ }^{2}$ en realidad han sido documentos y eventos de orden político, donde

2 Los informes son anuales, nunca remiten ni comparan sus resultados con series de varios años (por ejemplo, en los anexos de los Informes Presidenciales hay siempre una referencia a 20 años), por lo que no sirven como instrumento de referencia para evaluar las políticas emprendidas, pues se contentan con el recuento de los logros. 
los consejeros tenían un lugar secundario respecto de los miembros de la clase política local, donde no siempre se entregaban por escrito ni eran comparables entre sí; en los informes nunca es posible contrastar entre los objetivos planteados y los resultados realmente alcanzados, y casi siempre predomina el triunfalismo sobre la autocrítica.

El control burocrático y político de carácter autoritario al que estaban sometidos los universitarios es el verdadero adversario de la transparencia; ahí es a donde se trata de que llegue la luz y el escrutinio público, el propósito es terminar con la arbitrariedad y con el manejo discrecional de la información. Transparentar la información y alentar su utilización son las piezas clave de una nueva manera de ser universitario en el siglo XXI, porque ser parte de esta comunidad genera el compromiso de participar y contribuir colectivamente a la conducción institucional, a la consecución de los objetivos institucionales y al logro de las metas académicas que son el fundamento de la universidad.

\section{Las razones de la transparencia}

Entendemos por razones al conjunto de argumentos que justifican y dan sentido a la transformación jurídica; es decir, que intentan precisar los términos y propósitos buscados con la reforma legislativa. De esta manera, al precisar cuatro razones principales queremos contribuir a brindar elementos para exponer los motivos de la reforma y para tener claros algunos objetivos esperados que puedan ser evaluados en el futuro.

\section{La actualización del marco normativo}

La legislación que actualmente rige la vida institucional no contempla en ninguno de sus apartados la necesidad de la transparencia en las acciones ni mucho menos la rendición de cuentas ante la sociedad. Por tanto, la primera razón es, indudablemente, la actualización del marco normativo de la Universidad, y comenzar por la transparencia representa una oportunidad excepcional para perfilar el rumbo de su modernización legislativa.

El Consejo Universitario, al diseñar reformas que colocan a la Universidad ante un escenario que busca mejorar sus procesos, transparentar su operación y dotarse de instrumentos para la defensa de los derechos de los universita- 
rios, estableció los acuerdos para crear el Reglamento de Transparencia, el Ombudsman Universitario y el Consejo Asesor para el Abogado General. En el mismo sentido, comenzó a perfilar que el rumbo de la modernización legislativa de la Universidad debe orientarse para que ésta pueda ser capaz de sancionar, ${ }^{3}$ garantizar la defensa de los universitarios, afianzar la colegialidad de las decisiones, ${ }^{4}$ regular la relación jerárquica entre órganos, ${ }^{5}$ y establecer un gobierno universitario basado en un sistema de equilibrios entre los órganos colegiados y los órganos personales.

Específicamente, en lo que concierne al Reglamento de Transparencia, éste tendrá que ir, por lo menos, en concordancia con la Ley 848 de Transparencia y Acceso a la Información Pública para el Estado de Veracruz, teniendo además la obligatoriedad de atender la Ley Federal de Transparencia y Acceso a la Información Pública Gubernamental. Por lo anterior, la reforma de nuestro marco normativo es una obligación institucional irrenunciable, pues en el marco de la autonomía y dada la especificidad de la institución universitaria, si sólo nos atuviésemos a la referencia estatal o a la referencia de la Ley Federal, habría procesos y matices no considerados. ${ }^{6}$

Por lo demás, y de conformidad con lo establecido en el Programa de Trabajo 2005-2009 del rector de la Uv, entendemos que hay una voluntad expresa de construir una legislación de avanzada a nivel universitario, lo que implica conformar un marco normativo más radical en términos de transparencia, que abarque más ámbitos de los ya conquistados en otras legislaciones, que simplifique los procedimientos e incentive el uso y la consulta de la información. Necesitamos que desde el cambio legislativo se respalde la reforma universitaria y

3 En el caso de las computadoras salió a la luz el escaso instrumental jurídico, la ausencia de procedimientos, la indefinición de causales que hacen impreciso el establecimiento de sanciones.

4 En el mismo caso fue evidente que predomina un ejercicio fuertemente centralizado del poder, que da lugar a múltiples equivocaciones y problemas. En tanto organización de cultura superior, es indispensable el reforzamiento del poder colegiado y su control sobre los actos individuales.

5 Para garantizar que las autoridades deban escuchar y atender las recomendaciones y observaciones de los órganos colegiados, de los órganos de consulta o asesoría.

6 Por ejemplo, en un caso absurdo, la Ley estatal no nos obligaría a informar sobre la oferta académica en los diversos niveles de la Uv, estableciendo el modelo educativo, los horarios, los cupos, la modalidad educativa, los planes y programas de estudio indicando las fechas de aprobación, reforma y vigencia, las sedes donde se ofrecen, los profesores adscritos, así como información sobre los egresados y el mercado del empleo asociado a cada programa educativo. 


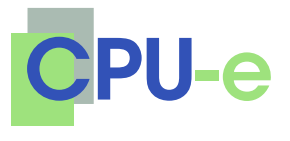

Razones por la transparencia en la universidad

Miguel Angel Casillas Alvarado

Jessica Badillo Guzmán

Karla Alejandra Valencia-González Romero

se imprima racionalidad a las políticas y acciones institucionales para que sean más eficientes y pertinentes. ${ }^{7}$

\section{La construcción de una nueva manera de ser universitario}

La democratización del régimen político debe también tener un efecto en la Universidad y en las relaciones entre los universitarios. Tal como sostiene Kent (2007), la construcción de una ciudadanía crítica universitaria es un imperativo del cambio institucional que supone la intervención de los universitarios en la gestión cotidiana de la institución, pues eso no sólo inhibe la corrupción o el dispendio, sino que genera adhesión a los objetivos institucionales y, por tanto, corresponsabilidad en su cumplimiento.

La participación de los universitarios es indispensable, pero para ello deben estar dotados de los insumos y de las disposiciones para usar la información. Debe haber, entonces, un proceso educativo al respecto, ya que requerimos de un cambio cultural, un cambio de mentalidades y, sobre todo, un cambio actitudinal ante la vida de nuestra institución. Lograrlo no será tarea fácil, pues históricamente la comunidad universitaria no ha formado parte activa en la toma de decisiones, pues suele no ser considerada para tal efecto; pero tampoco ha propugnado por defender este derecho y hacerlo observable. La mayoría de los actores universitarios, principalmente el sector estudiantil, atraviesa por la Universidad sin que sea plena su integración a la vida institucional.

Los universitarios muy pocas veces se incorporan a la discusión de los procesos administrativos y académicos de su Institución, tanto porque les son opacos, como por la falta de espacios y prácticas institucionales que lo promuevan. En muchas ocasiones, incluso, hay un desconocimiento de qué es lo que está ocurriendo en su Universidad.

Por lo tanto, es necesario fomentar en toda la comunidad universitaria el interés hacia la institución de la cual forma parte y el compromiso para con la misma. Pero también hay que transformar las estructuras y las prácticas de la gestión institucional, ampliando los espacios de participación de académicos y estudiantes, incrementando los niveles de intervención de los órganos colegiados y

7 Al ampliar los ámbitos de información que debe hacer pública, la institución se obliga a producir dicha información, y por tanto se coloca en posición de observar problemas y características no reconocidos anteriormente, teniendo entonces elementos para emprender acciones correctivas. 
los órganos de asesoría, impulsando el debate en los medios de la Universidad. Así también, es prioritario que los universitarios adopten una postura crítica en relación a la Universidad, sus acciones y los resultados que de éstas se deriven; se requiere un amplio proceso formativo y de difusión que contemple el derecho que la comunidad universitaria tiene de conocer la vida institucional en todos sus ámbitos, pero además, ponga de manifiesto la obligación de ejercer tal derecho en un acto responsable y ético, que sirva de control para el actuar de las autoridades, funcionarios y personal que labora en la Universidad.

El ejercicio de una postura crítica por parte de la comunidad universitaria, implica alentar que se denuncien y puedan ser denunciadas las arbitrariedades, atropellos o violaciones a la legalidad de la institución; implica además que los universitarios orienten sus votos para nombrar representantes o autoridades teniendo elementos objetivos de información sobre su desempeño, honorabilidad y capacidad académica. Por último, implica que los universitarios cuenten con los datos y los elementos de juicio para poder ejercer la crítica, que es uno de los valores académicos trascendentes y fundamento mismo de la institución universitaria.

La conformación de usuarios responsables tiene entonces un doble impacto en la Universidad. Por un lado, se convierte en un instrumento para la evaluación de los procesos institucionales, educativos y cotidianos de la Universidad. Pero por otro lado, es la mejor manera de contender contra el uso político y sesgado de la información, puesto que frente a la difamación y la calumnia, frente al uso tendencioso o sensacionalista, frente a la diatriba, no hay mejor herramienta que una comunidad cohesionada en torno a su proyecto institucional, comprometida con el desarrollo académico y con los valores éticos que norman la vida de los universitarios.

\section{Transparencia, autonomía y rendición de cuentas a la sociedad}

Las universidades en general, son depositarias de la confianza pública, pues en ellas concurren e imponen sus leyes los eruditos y los sabios (Kant, 2002; Bourdieu, 2002). La sociedad tiene la confianza de que ahí se forman los profesionistas y los científicos, que en ese tipo de instituciones se produce conocimiento científico de una manera rigurosa, que desde ahí se difunden las artes y las ciencias. Las universidades, en tanto instituciones sociales, han sido conferidas de cumplir con excelencia sus funciones, y deben rendir cuentas de cómo lo hacen. 
Con mayor razón deben rendir cuentas si se trata de entidades públicas, financiadas con los recursos de los contribuyentes. La Universidad Veracruzana, en su carácter de organismo autónomo, tiene la obligación de servir al país con eficiencia, eficacia y transparencia, debiendo informar con calidad, veracidad y oportunidad sobre su objeto, el desarrollo de sus fines y de sus funciones sustantivas, además de rendir cuentas sobre los recursos que le son destinados, haciéndolos del conocimiento de la comunidad universitaria y de la sociedad en general.

La autonomía universitaria, lejos de ser un impedimento o un pretexto para que la Universidad oculte la información que de ella se genera, debe ser un instrumento que permita someter al escrutinio público sus acciones y los resultados de las mismas. A mayor y mejor información, mayor escrutinio público, mayor confianza sobre nuestras acciones, y por tanto, mayor legitimidad social para justificar nuestra existencia y para encontrar apoyo cuando se trate de solicitar incrementos presupuestales. La autonomía depende de la confianza pública, su ejercicio es fundamental para producir conocimientos y enseñar con libertad; fortaleciendo entonces la legitimidad social, incrementamos nuestra libertad académica.

\section{La contribución de la Universidad a la formación de ciudadanos}

Como se ha venido sosteniendo, la transparencia y el acceso a la información se han vuelto aspectos primordiales de nuestro presente y nuestro futuro nacional, en la construcción de una sociedad democrática y abierta. Con el surgimiento de la transparencia y acceso a la información en la agenda pública nacional, las universidades se configuran como el espacio ideal para la formación de una ciudadanía responsable, democrática y crítica, consciente de que el derecho a la información deriva en una efectiva rendición de cuentas por parte de las instituciones.

Como Pablo Latapí (2007) reconoce:

Las universidades no existen sólo para crear y promover el conocimiento económicamente útil, sino todas las formas de conocer que requiere la sociedad. Pues la universidad es una institución responsable de generar, proteger y difundir todos los tipos de conocimiento que requiere el país, también los aparentemente improductivos.

Lo anterior va muy ligado al concepto de función social de las universidades que desde hace unos años se viene incluyendo en el discurso educativo y, más aún, en el contexto de la emergente sociedad del conocimiento, en cuyo desarrollo se ha asig- 
nado a la universidad un papel fundamental. La Universidad Veracruzana, desde su proyecto institucional, ha establecido la importancia de su función social y de la producción de conocimiento para su distribución hacia la sociedad, definiendo como prioridad la formación de ciudadanos con una nueva perspectiva de desarrollo, no sólo económico, sino intelectual, ambiental, cultural y científico.

Sumado a lo anterior, en el contexto de la transparencia, la Universidad Veracruzana adquiere nuevos compromisos y requiere, por lo tanto, nuevas acciones.

La Universidad debe contribuir a la formación de ciudadanos en la democracia. La promoción de la democracia, para ser efectiva, deberá ir más allá del mero discurso, debiendo incorporarse a las prácticas y a las actividades institucionales. De esta manera, la promoción de la democracia puede cristalizarse en la vida universitaria fomentando la participación de la comunidad, por ejemplo, en la elaboración de leyes y reglamentos, enseñando a legislar y a debatir, a elaborar propuestas que sean presentadas y discutidas; educar al ciudadano universitario a participar en este proceso, coadyuvará a que éste pueda participar de manera activa en la vida social, responsabilidad que deberá ser compartida por autoridades educativas y académicos.

Una institución universitaria que desarrolla prácticas democráticas (deliberación razonada, definición de acuerdos sin coacciones, implementación de acciones de manera colectiva, en el marco del ejercicio pleno de libertades de expresión y manifestación) es una institución que está formando a sus estudiantes en la cultura política democrática que como sociedad hemos definido.

La formación de ciudadanos también implica la intensificación del debate político y el incremento tanto de los espacios como de los interlocutores. Esto es, la Universidad, para avanzar en su proceso de democratización, deberá reformar sus instrumentos de comunicación (para abrirlos a la comunidad) y ampliar su cobertura, hasta convertirlos en recursos que contribuyan cotidianamente al debate institucional.

La ampliación de las redes ciudadanas pasa por el manejo y uso de la información, de ahí que la Universidad contribuye a la democracia cuando decodifica información, produce conocimiento y lo pone en relación con los diferentes agentes sociales. La única capacidad social de controlar el sofisticado lenguaje tecnocrático descansa en que tanto los egresados universitarios como la sociedad en general (a través del cumplimiento de la divulgación científica) sean capaces de comprenderlo y decodificarlo, para poder fundamentar una posición crítica.

Con el pleno ejercicio de sus funciones, la Universidad contribuye al desarrollo social; hablando de democracia (Fuentes, I989), participa formando ciu- 


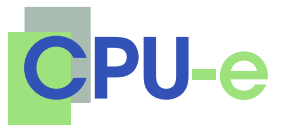

Razones por la transparencia en la universidad

Miguel Angel Casillas Alvarado

Jessica Badillo Guzmán

Karla Alejandra Valencia-González Romero

dadanos comprometidos con valores y conocimientos socialmente pertinentes, con habilidades de pensamiento crítico que permitan poner en cuestión los discursos e informes públicos, con capacidad para trabajar con grandes volúmenes de información y con un conocimiento experto sobre los problemas naturales y sociales. Con la investigación científica la universidad contribuye produciendo conocimiento y con su divulgación se fortalecen las capacidades de la sociedad para diagnosticar con precisión y para diseñar alternativas a sus problemas.

En su sentido axiológico, la Universidad Veracruzana debe promover una serie de valores entre los cuales se inscribe, hoy, la transparencia. La formación de ciudadanos alrededor de la valorización de la transparencia, en su aplicación y en su defensa, deberá ser una prioridad más en la vida universitaria, pues una vez que los estudiantes egresen y se incorporen a la sociedad productivamente activa, requerirán de un alto sentido de responsabilidad, de justicia, de equidad y tolerancia, pero también de transparencia, crítica y autocrítica hacia sus acciones y hacia las de los demás.

Por último, la Universidad deberá contribuir a la formación de ciudadanos en relación al acceso a la información. Por siglos, muchas cuestiones de la vida social (e incluso institucional) se han acatado como "secretas", como espacios inviolables que controlan unos cuantos. En esta nueva era del conocimiento, se requiere de hombres y mujeres que exijan su derecho a conocer, por lo que resulta fundamental que desde las universidades se dote a la ciudadanía de las herramientas conceptuales y prácticas necesarias para hacer un uso eficiente de su derecho a la información, a través de sus mecanismos de generación y distribución de conocimiento.

No hemos querido apuntar propiamente una conclusión, pues, como hemos señalado anteriormente, la Transparencia Universitaria no es un asunto cerrado, sino un elemento que debe seguir construyéndose día a día en el quehacer institucional, cuya edificación debe tomar lugar en todos los espacios y sustentarse en todos los actores. Por tanto, sirvan estas líneas para dejar en claro que la Universidad tiene principalmente cuatro razones para la transparencia: la actualización del marco normativo; la construcción de una nueva manera de ser universitario; la relación entre transparencia, autonomía y rendición de cuentas a la sociedad; así como su contribución a la formación de ciudadanos. Alrededor de estas razones deberá configurarse la exposición de motivos para la reforma universitaria en materia de transparencia, motivos que serán la base para la evaluación de las acciones emprendidas y por emprender en este mismo ámbito. 
Miguel Angel Casillas Alvarado Jessica Badillo Guzmán

Karla Alejandra Valencia-González Romero

\section{Lista de referencias}

Kent, R. (2007). "La transparencia en las universidades: el derecho a la información, la modernización irreflexiva y la claudicación de la pedagogía crítica”. Ponencia presentada en el Encuentro sobre la Transparencia y el Acceso a la Información en las Universidades Públicas, convocado por el IfAI-Uv, I5 y I6 de marzo de 2007, Xalapa, Ver.

Bourdieu, P. (2002, enero-diciembre). El conflicto de las facultades. Colección Pedagógica Universitaria, 37-38, pág. 43-84. (Traducción de Miguel Casillas).

Kant, I. (2002, enero-diciembre). El conflicto de las Facultades. Colección Pedagógica Universitaria, 37-38, pág. 85-I06. (Traducción de Miguel Casillas).

Latapí, P. (2007). Conferencia Magistral al recibir el Doctorado Honoris Causa de la Universidad Autónoma Metropolitana. México, D. F.

Fuentes, O. (I989). "Universidad y Democracia en México: La mirada hacia la izquierda." Contribuciones Programa FLACSO-Chile. Núm. 59. dentro de la Serie "Transformaciones de los sistemas de educación superior, realizado por la FLACSO con el apoyo del International Development Research Center. 